\title{
3D Imaging of Non-spherical Silicon Nanoparticles Embedded in Silicon Oxide by Plasmon Tomography
}

\author{
Aycan Yurtsever*, Matthew Weyland*, David A Muller* \\ * School of Applied and Engineering Physics, Cornell University, Ithaca, NY 14850
}

Silicon nanoparticles embedded in silicon oxide are a promising silicon-compatible material for integrated photonics [1] and memory [2] devices. This system shows room-temperature photoluminescence in the visible band, net optical gain and luminescence at the telecommunications band of $1.54 \mu \mathrm{m}$ when doped with Er. Although extensive research has been carried out with respect to potential device applications, microstructural characterization has proved more challenging due to the complexity of the material. Two-dimensional (2D) energy-filtered TEM studies suggest the optimal size distribution in photonic applications occurs when the average particle size is less than $3 \mathrm{~nm}$ [3]. At typical sample thicknesses (10-50nm) and densities the nanoparticles are too closely spaced to distinguish between isolated particles or networks or even reliably determine their morphology. The purpose of this study is to resolve these problems by nano-scale plasmon loss tomography and provide a means to measure the nanoparticle shape and distribution in all three dimensions. This is critical for determining the transport and injection mechanism (tunneling vs. impact ionization) for optical excitations and electron storage.

A tilt series of plasmon images was acquired for $\mathrm{Si}$ at $17 \mathrm{eV}$ with $4 \mathrm{eV}$ wide energy window, from $\pm 60^{\circ}$ at $4^{0}$ increments to limit the does. Alignment and reconstruction of the tilt series was carried out using scripts programmed in IDL V5.6. Fig. 1a. shows the reconstruction of Si plasmon loss images visualized by volume rendering and an iso-surface and fixed threshold. Due to the reduction of the bulk plasmon signal in the smaller particles $(<6 \mathrm{~nm})$, a single iso-surface tends to miss smallest particles, thus underestimate particle density. Volume rendering, on the other hand, provides a complete chemical map of Si distribution, although distinguishing particles in projection is difficult. Therefore, iso-surfaces must be interpreted together with volume rendering when size, density and shape of the particles are analyzed. Our result in Fig. 1. demonstrates that most particles have complex morphologies and not simple spherical symmetry.

A more detailed view of one nanoparticle is shown in Fig. 2. A projection of the particle (Fig. 2a) shows features that would probably be identified as two separate spherical particles if this were the only information provided. However, the tomographic reconstruction reveals this to be a single particle with a "horseshoe" morphology (Fig. 2b,c). This unusual shape has direct consequences for the wave functions of electron-hole pairs formed in the particle. In addition, the higher surface to volume ratio $(0.55)$ than the sphere of equivalent volume $(0.35)$, would mean a higher interface density of states, increasing their importance for excitation lifetime and recombination. Both of these electronic structure predictions would significantly affect optical properties of this material.

We have shown that Si nanoparticles embedded in silicon oxide do not have simple spherical symmetry; instead complex morphologies with high surface to volume ratios were observed. The lower symmetry structures would have less degeneracy in their energy levels than simple spherical particles, and so a broader distributions of sub-bands in each of the quantum dots. When further 
averaged over many randomly oriented particles, this could explain the observed broad photoluminescence emission band [4].

\section{References}

[1] L. Pavesi, L. D. Negro, C. Mazzoleni, G. Fronzo, and F. Priolo, Nature 408, 441 (2000)

[2] S.Tiwari, F. Rana, H. Hanafi, A. Hartstein, and E. F. Crabbe, Appl. Phys. Lett. 68, 1377 (1996)

[3] F. Iacona, C. Bongiorno, C. Spinella, S. Boninelli, and F. Priolo, J.Appl.Phys. 95, 3723 (2004).

[4] This work is supported primarily by the Nanoscale Science and Engineering Initiative of the National Science Foundation under NSF Award \# EEC-0117770
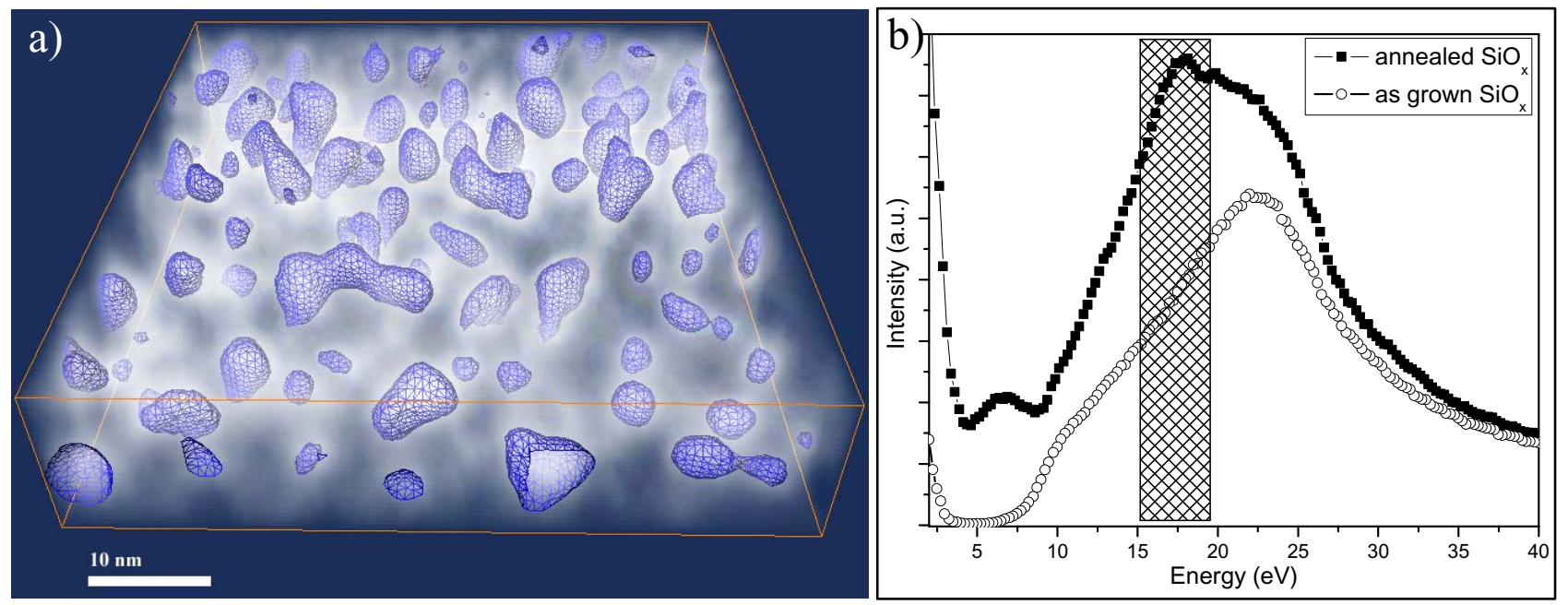

FIG. 1. a) Tomographic reconstruction of Si plasmon signal at $17 \mathrm{eV}$, visualized by volume rendering (white "fog") and an iso-surface at fixed threshold (blue shapes). Complex, non-spherical morphologies are dominant. b) Low-loss electron energy loss spectra for annealed and as-grown $\mathrm{SiO}_{\mathrm{x}} .4 \mathrm{eV}$ wide energy filter was placed on bulk Si plasmon signal (rectangle). Spectra were shifted for clarity.
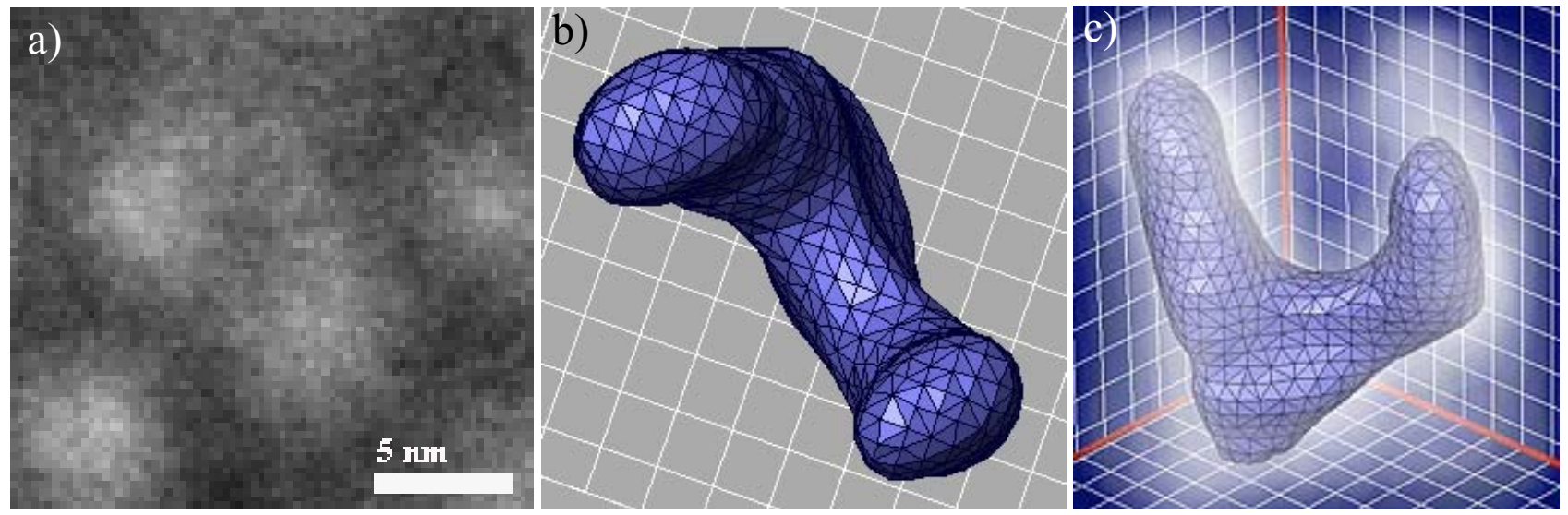

FIG. 2. a) Zero tilt plasmon loss image, b) top view and c) side view of the reconstructed nanoparticle. Projection a), shows features that would probably be identified as two separate spherical particles. However, 3D reconstruction reveals this to be a single particle with a "horseshoe" morphology b) and c). (Edge of the square grid is $1 \mathrm{~nm}$., white "fog" in c) is volume rendering of bulk Si plasmon signal, particle is visualized by surface rendering in b) and c)). 